\section{SP6-53 ASSOCIATIONS BETWEEN BIRTH WEIGHT, GENDER AND TEETH ERUPTION IN INFANTS IN ARKHANGELSK, NORTHWEST RUSSIA}

doi:10.1136/jech.2011.142976q.24

\begin{abstract}
${ }^{1,2} \mathrm{M}$ Gorbatova, ${ }^{2} \mathrm{~L}$ Gorbatova, ${ }^{1,2} \mathrm{M}$ Pastbin, ${ }^{2} \mathrm{~T}$ Ushmanova, ${ }^{3,4} \mathrm{~A}$ Grjibovski. ${ }^{* 1}$ International School of Public Health, Northern State Medical University, Arkhangelsk, Russia; ${ }^{2}$ Northern State Medical University, Arkhangelsk, Russia; ${ }^{3}$ Norwegian Institute of Public Health, Oslo, Norway; ${ }^{4}$ nstitute of Community Medicine, University of Tromsø, Tromsø, Norway
\end{abstract}

Introduction Birth weight is associated with many health-related conditions. However, associations with dental health remain largely unknown. Infants in Russia have on average eight erupted teeth by the age of 48 weeks. We studied associations between birth weight and eruption of deciduous teeth in Arkhangelsk, Northwest Russia. Methods Altogether, 456 randomly selected infants (51.3\% were boys) at the age of 11-13 months (mean 47.8 weeks) underwent dental examination in a paediatric clinic in the city of Arkhangelsk. Data on birth weight were obtained from medical records. Mean numbers of erupted teeth by gender were compared using Mann-Whitney tests. Associations between having eight or more teeth and birth weight (in kilogrammes) were assessed by logistic regression with adjustment for gender, infant age and breastfeeding history.

Results The mean number of erupted teeth was 6.69 (95\% CI 6.47 to 6.91). It was higher in boys than in girls (7.04 vs $6.32, p=0.001)$. Altogether, $43.6 \%$ (49.8\% of boys and $37.0 \%$ of girls) of all examined children had 8 or more teeth. A positive association between birth weight and having 8 or more teeth was observed: OR 1.79 (95\% CI 1.19 to 2.70 ). Girls were less likely to have 8 or more teeth than boys: OR 0.65 ( $95 \%$ CI 0.43 to 0.98 ).

Conclusions The mean number of erupted teeth in infants in Arkhangelsk is lower than in Russia on average. Birth weight and male gender were positively associated with the odds of having 8 teeth by the age of 48 weeks. Potential mechanisms will be discussed.

\section{SP6-54 SUGGESTIVE BEHAVIOURS OF EATING DISORDERS IN UNDERGRADUATE FEMALE STUDENTS: PREVALENCE AND ASSOCIATED FACTORS}

doi:10.1136/jech.2011.142976q.25

\begin{abstract}
${ }^{1} V$ Magalhães, ${ }^{*}{ }^{1,2} \mathrm{C}$ Meneses, ${ }^{1,2} \mathrm{G}$ Mendonça. ${ }^{1}$ Universidade do Estado do Rio de Janeiro, Rio de Janeiro, Brazil; ${ }^{2}$ Centro Universitário Celso Lisboa, Rio de Janeiro, Brazil
\end{abstract}

Eating disorders are behavioural syndromes which diagnoses criteria have been widely studied during the last decades. Anorexia nervosa, bulimia nervosa and partial syndromes stand out. Metabolic and electrolyte abnormalities and endocrine alterations can be present. Early diagnosis and an adequate management of these complications are important to reduce the risks associated. The objective of the study was to assess the prevalence of suggestive behaviours of eating disorders and associated factors, in undergraduate female students from a public university in the city of Rio de Janeiro. Cross-sectional study was conducted in 2004. The sample consisted of 512 students who answered a self-reported questionnaire based on the Eating Attitudes Test (EAT-26), Bulimic Investigatory Test of Edinburgh (BITE), used to investigate behaviours suggestive of disorders. It also included the General Health Questionnaire and questions about body image perception, leisure-time physical activity and nutritional evaluation. Ratios prevalences were estimated through Poisson regression. The mean age was 21.5 years old. Prevalences of $16.99 \%$ have been detected in the EAT-26, 25.00\% in the BITE, and $33.40 \%$ in the joint analysis of both instruments. There was significant association between behaviour suggestive of eating disorders and attending a course in the field of Education and Humanities (PR 1.41; 95\% CI 1.06 to 1.89), age between 17 and 20 years (PR 1.78; 95\% CI 1.01 to 3.14 ), and between 20 and 30 years old (PR 1.75; 95\% CI 1.01 to 3.04), self-perception of being overweight (PR 1.84; 95\% CI 1.39 to 2.42), and presence of common mental disorder (PR 1.42; $95 \%$ CI 1.01 to 1.83 ). These results showed that educational actions for control and prevention of eating behaviour suggestive of disorders among undergraduate female must be fostered.

\section{SP6-55 SEXUAL DEVELOPMENT AND LUNG FUNCTION IN ADOLESCENTS AGED 15 YEARS OLD: THE 1993 PELOTAS BIRTH COHORT}

doi:10.1136/jech.2011.142976q.26

F C Wehrmeister, * J Martínez-Mesa, R B Noal, P R C Hallal, C L P Araújo, H Gonçalves, A M B Menezes. Federal University of Pelotas, Pelotas, Rio Grande do Sul, Brazil

Background Lung function, measured by $\mathrm{FEV}_{1}$ and FVC, increases with age and height and it has an accelerated increase during adolescence.

Objective To describe the relation between sexual development and lung function at the age of 15 in a birth cohort.

Method All children $(\mathrm{N}=5249)$ born in the city of Pelotas, Brazil, were enrolled in a prospective birth cohort. In 2008, all cohort members were sought for a follow-up visit (response rate $=85.7 \%$ ). Sexual development was obtained using Tanner stages in five categories (from 1 to 5). VEF1 and FVC were measured in litres by spirometry and used as continuous variables. A multiple linear regression model stratified by sex and adjusted for family income, mother's height and wheezing, adolescent's height, age, smoking, and skin colour was performed. For girls it was also adjusted by age of menarche.

Results In boys, sexual development was not associated with $\mathrm{FEV}_{1}$ or FVC. On the other hand, among girls the regression coefficients for $\mathrm{FEV}_{1}$ and Tanner stages 2, 3, 4 and 5 were, respectively: $\beta=0.07$ (95\% CI -0.04 to 0.18 ), $\beta=0.13$ (95\% CI 0.02 to 0.24$), \beta=0.15$ ( $95 \%$ CI 0.04 to 0.26 ), $\beta=0.14$ (95\% CI 0.02 to 0.27 ). The regression coefficients for FVC and Tanner stages $2,3,4$ and 5 were, respectively: $\beta=0.11$ (95\% CI -0.03 to 0.24$) ; \beta=0.21$ (95\% CI 0.08 to 0.33 ); $\beta=0.25$ (95\% CI 0.12 to 0.38 ) and $\beta=0.23$ (95\% CI 0.09 to $0.38)$.

Conclusion Sexual development is associated with lung function among girls but not boys, probably due to the earliest sexual development in girls.

\section{SP6-56 ARE MALES REALLY AT GREATER RISK FOR DOG BITES THAN FEMALES?}

doi:10.1136/jech.2011.142976q.27

L Messam, ${ }^{*}$ H Y Weng. University of California Davis, Davis, California, USA

Introduction It is received wisdom that males are more likely to be bitten by a dog than females. While this is consistent with a greater perceived tendency towards risk-taking behaviour in males, previous studies investigating the gender-dog bite relationship have been limited either by a lack of or an improperly chosen comparison group.

Methods To investigate whether males are at higher risk for dog bites than females, we conducted a hospital-based case-control study in Kingston, Jamaica. Cases were all (120) dog bite victims (56 males, 64 females) seen in the outpatient department of St. Andrews Memorial Hospital in Kingston, from 1 January 2002 to 30 June 2003. Three control groups were selected from among outpatients 